\title{
Rabaska
}

Revue d'ethnologie de l'Amérique française

\section{Regroupement des étudiants(e)s des programmes d'ethnologie de l’Université Laval (RÉPEUL)}

\section{Jocelyn Gadbois}

Volume 3, 2005

URI : https://id.erudit.org/iderudit/201747ar

DOI : https://doi.org/10.7202/201747ar

Aller au sommaire du numéro

Éditeur(s)

Société québécoise d'ethnologie

ISSN

1703-7433 (imprimé)

1916-7350 (numérique)

Découvrir la revue

Citer ce document

Gadbois, J. (2005). Regroupement des étudiants(e)s des programmes d'ethnologie de l'Université Laval (RÉPEUL). Rabaska, 3, 214-215.

https://doi.org/10.7202/201747ar d'utilisation que vous pouvez consulter en ligne. 


\section{Regroupement des étudiants(e)s des programmes \\ d'ethnologie de l'Université Laval (RÉPEUL)}

Pavillon Charles-De Koninck

Courriel : repeul_exe@hotmail.com

Local 0221, Université Laval

Québec (Québec) G1K 7P4

L'année 2004-2005 fut bien remplie pour le Regroupement des étudiant(e)s des programmes d'ethnologie de l'Université Laval. Le comité de direction était formé cette année de Jocelyn Gadbois (président), de Marc-André Lavoie (vice-président aux affaires internes et externes), de Francesca Désilets (viceprésidente aux activités socioculturelles et aux communications), de MarieÈve Gravel (trésorière) et de Frédérique Cornellier (secrétaire). Marc-André Lavoie a dû quitter son poste avant la fin de son mandat puisqu'il a été élu vice-président à l'enseignement et à la recherche au sein du bureau de la CADEul. Les membres du Répeul tiennent à le féliciter pour son excellent travail. La secrétaire, Frédérique Cornellier, a été élue pour le remplacer. Mandoline Langlois, représentante à la pédagogie, mérite également les félicitations des membres pour avoir maintenu avec brio l'évaluation formative des cours d'ethnologie pendant une deuxième année consécutive. Cette année, le travail acharné des officiers du RéPEuL leur a permis d'organiser maintes activités (initiation, soirées, escapades, mobilisation, etc.), de se stabiliser financièrement, de réorganiser ses communications (le bulletin d'ethno revit maintenant), et surtout de prêter sa voix à nombre de débats, de sujets et de projets au sein de la communauté universitaire.

En plus de ses présences remarquées au sein des caucus de la CADEUL, il a multiplié ses interventions dans diverses sphères (Faculté des lettres, comités de programme, d'accueil, de mobilisation, groupe de réflexion sur le partenariat entre le Musée de la civilisation et l'Université Laval, etc.). Le RÉPEUL a cherché à sensibiliser le département d'Histoire au manque flagrant de professeurs en ethnologie. Il a solidifié le sentiment d'appartenance aux programmes d'ethnologie, a fait la promotion desdits programmes par le biais de documents promotionnels qu'il a lui-même créés, a offert des livres des finissants aux premiers diplômés du baccalauréat intégré, et bien d'autres.

Le bilan de l'année 2004-2005 ne saurait être complet sans mentionner le mandat de grève générale qui a été en vigueur du 16 mars au 4 avril 2005 afin de revendiquer l'abolition de la réforme de l'Aide financière aux études (les coupures de $103 \mathrm{M} / \$$ ) et l'abolition de la réforme des cégeps. Le RÉPEUL s'est ainsi investi corps et argent dans diverses activités de mobilisation en plus d'un piquetage devant chaque classe d'ethnologie comme des manifestations, des bed in, des lettres ouvertes, etc. Or, même si les membres 
du RÉPEUL ont mis un terme à cette grève, ils ont refusé à l'unanimité l'offre du ministre Fournier.

La prochaine année s'annonce également mouvementée, notamment avec l'organisation de la prochaine initiation, la poursuite et le maintien de certains dossiers et, qui sait, un boycottage plus structuré contre Sodexho.

JOCELYN GADBOIS 